\title{
Platelets in rheumatoid arthritis: exploring the anti-inflammatory and antithrombotic potential of TNF inhibitors
}

\author{
Armen Yuri Gasparyan, ${ }^{1}$ George D Kitas ${ }^{1,2}$
}

Manfredi et $a l^{1}$ explore the relationship between tumour necrosis factor $\alpha(\mathrm{TNF} \alpha)$, TNF $\alpha$ inhibition and platelet activation in rheumatoid arthritis (RA). Using elegant systems they demonstrate that $\mathrm{TNF} \alpha$ activates platelets and promotes their proinflammatory and procoagulant actions, while TNF inhibition (TNFi) prevents platelet activation. They suggest this is a potential mechanism explaining why TNFi associates with a reduction of cardiovascular (CV) events in patients with RA, as shown in some observational studies. Should we then be(come) interested in platelet biology in RA?

The potential role of platelets in the pathogenesis of RA and related comorbidities has, in fact, received much less attention than it deserves. Platelets are small $(1-2 \mu \mathrm{m})$ anucleate cells that are intimately involved in thrombosis, angiogenesis, bone remodelling, inflammation and autoimmunity. Their bidirectional, damaging and protective roles across pathophysiological processes have been explored in several clinical and laboratory studies. The discovery of specific platelet markers and agonists, which target a range of membrane-bound receptors, has resulted in better understanding of their role. It is now clear that platelet reactivity throughout their short lifespan (8-10 days) is determined by megakaryopoiesis, which is regulated quantitatively by thrombopoietin; any chemical compound targeting the maturation of platelet precursors in the bone marrow either directly or indirectly can alter the thrombotic, immune and inflammatory potential of circulating platelets. ${ }^{2} 3$

Activated platelets shed highly active membranous structures-microparticlesand transform from disc-shaped to enlarged cells with pseudopods that facilitate their interaction with other

\footnotetext{
${ }^{1}$ Departments of Rheumatology and Research and Development, Dudley Group NHS Foundation Trust, Russells Hall Hospital, Dudley, UK; ${ }^{2}$ Arthritis Research UK Epidemiology Unit, University of Manchester, Manchester, UK

Correspondence to Professor George D Kitas, Research and Development Directorate, Dudley Group NHS Foundation Trust, Russells Hall Hospital, Dudley DY1 2HQ, UK; kitas@dgh.nhs.uk
}

platelets, neutrophils, lymphocytes and other immune cells. Circulating neutrophils, in turn, interact with activated platelets to boost their 'thromboinflammatory' potential. ${ }^{4}$ Circulating cellular complexes of platelets are often found in the blood stream of patients with inflammatory conditions, such as RA, and it is suggested that platelet-derived microparticles along with other biological agents play a more important role in the development of synovitis than activated platelets themselves. ${ }^{5}$ Indeed, laboratory studies have demonstrated that platelets exert arthritogenic properties through the activation of the collagen glycoprotein VI receptor and subsequent shedding of microparticles. ${ }^{6}$

As effector cells derived from megakaryocytes, platelets bear a wide variety of membrane receptors of their precursors, autonomous protein-synthesising platform (messenger RNA) and the granular system of accumulated biologically active compounds (ie, $\alpha$ granules, dense granules, lysosomes) facilitating their involvement in diverse immune reactions. $\alpha$ Granules are especially rich in inflammatory cytokines, vasoactive substances, chemokines and stimulators of platelet aggregation, which are abundantly released upon platelet activation. Biological agents of platelet granules propagate inflammation, increase vascular permeability and destroy cells at inflamed sites. Such processes could be involved in the initiation and propagation of the inflammatory response and tissue destruction both in the rheumatoid joint and the vasculature and merit further investigation in RA. At exactly the opposite end, experimental and clinical studies have shown that some components of autologous platelet-rich plasma, and particularly ADP of dense granules, are capable of stimulating proliferation of chondrocytes and deposition of collagen I, thereby repairing damaged cartilage and tendons. ${ }^{78}$ There is, therefore, a distinct possibility of regulating various pathophysiological processes in joints and surrounding tissues by suppressing and activating platelets at different stages of the disease.

A variety of specific markers of platelet activation have been tested across inflammatory rheumatic diseases: platelet count, membrane- bound P-selectin and CD40 ligand (CD40L), $\beta$-thromboglobulin, platelet factor 4 (PF4), also known as chemokine (C-X-C motif) ligand 4 (CXCL4), platelet aggregates and platelet-neutrophil complexes. ${ }^{5}$ Reactive thrombocytosis with elevation of platelet count has been suggested to be of diagnostic importance in the setting of antineutrophil cytoplasmic antibodies-associated vasculitis. ${ }^{9}$ Mildly elevated platelet count along with decreased platelet size have been viewed as a reflection of inflammatory megakaryopoiesis and activity of RA, ${ }^{10}$ while the ratio of platelets to neutrophils and lymphocytes has also been suggested to reflect rheumatoid activity. ${ }^{11}$ A recently emerged important piece of evidence points to the fact that anticitrullinated protein antibodies (ACPA) directly stimulate the expression of P-selectin on platelets, secretion of soluble CD40L and formation of platelet aggregates. ${ }^{12}$ With the incremental increase of ACPA preceding clinically manifest RA by months and years, it is possible that insidious seropositivity and increase of C-reactive protein determine platelet activation and involvement from the earliest stages of synovial inflammation onwards.

In the era of biological therapy several lines of evidence suggest that agents inhibiting TNF $\alpha$ and interleukin- 6 suppress disease activity and decrease platelet counts in RA. ${ }^{13} 14$ The effect of biologic diseasemodifying antirheumatic drugs (bDMARDs) on platelet counts is not matched by nonbiologic DMARDs, which appear to be less effective towards megakaryopoiesis. The exception is bone marrow suppression and related thrombocytopenia as a rare occurrence of sulfasalazine therapy, ${ }^{5}$ which however is no longer a frontline choice in RA. Interestingly, the possible effects of bDMARDs on megakaryopoiesis may differ, with reports suggesting that tocilizumab may drop platelet counts in patients with RA more potently than adalimumab. ${ }^{15}$ This is another area that requires further investigation, as a potential side effect and also as a possible desirable effect. IL-6 is known as a physiological regulator of megakaryopoiesis acting likes thrombopoietin, ${ }^{16}{ }^{17}$ and blocking IL-6-related pathways may lead to antiinflammatory and antithrombotic effects.

The present flow cytometric and electron microscopy study by Manfredi et al, ${ }^{1}$ sheds light on the molecular basis of modulation of platelet activity by inflammatory cytokines and their inhibitors. The authors should be congratulated for their extensive work characterising the effects of recombinant human TNF $\alpha$ and the TNFi, infliximab, on platelet-rich plasma and isolated platelets of patients with RA (box 1).

One of the important messages of this study is that TNFi can only block the TNF $\alpha$-dependent activation pathways of 


\section{Box 1 New knowledge and limitations of the study by Manfredi et al ${ }^{1}$}

\section{Important new data}

- Tumour necrosis factor $\alpha(\mathrm{TNF} \alpha)$ depletes $\alpha$ granules of rheumatoid platelets (TNF $\alpha$ receptor 1-mediated effect).

- TNF $\alpha$ leads to the expression of P-selectin, CD40L, tissue factor, fibrinogen binding to rheumatoid platelets and forming platelet-neutrophil complexes (TNF $\alpha$ receptor 2-mediated effect).

- Infliximab abrogates TNF $\alpha$-specific platelet-activating effects but does not affect several other activation pathways in rheumatoid platelets.

- Platelets in rheumatoid arthritis (RA) are more activated than in coronary artery disease and osteoarthritis.

Limitations

- The relationships between platelet markers and cardiovascular risk/comorbidities in RA were not explored.

- Platelet count, a proxy of inflammatory megakaryopoiesis, was not analysed in relation to flow cytometric markers of platelet activation in RA.

platelets. Other pathways of platelet activation, particularly those regulated by collagen and ADP, can still continue contributing to proinflammatory and prothrombotic actions. Although platelet count was not analysed in connection with flow cytometric markers, and this can be viewed as a limitation, it is plausible that TNFi exert their effects on platelet receptors and also on platelet precursors maturing throughout megakaryopoiesis.

The authors have concentrated almost exclusively on the concept of CV comorbidity and its reduction in RA. However, the mechanisms they have described may be as important in the very essence of the disease, joint inflammation per se. It is, therefore, slightly disappointing not to see the wealth of data generated, analysed in the context of disease activity, using well-established clinical and laboratory indices.

To return to the original thesis: has this study answered the question as to whether inhibition of platelet activation by TNFi translates into CV protection in RA? As the authors themselves point out it has not, due to evident design limitations such as its crosssectional nature, small sample size, lack of long-term longitudinal follow-up and inability to control for multiple potential confounders (box 1). The study is a good reference for future studies aiming to address $\mathrm{CV}$ risk reduction in RA. The CV implications of antirheumatic therapies have gained almost as much importance as their primary efficacy and safety targets but studies to address them are extremely difficult to conduct. ${ }^{18}$ Very large numbers, longitudinal design, the ability to control for multiple $\mathrm{CV}$ and other confounders that may be changing over time, and very specific, ideally hard endpoints are required as a minimum. Potential difficulties should be foreseen and accounted for. For example, longitudinal studies addressing platelet biology may be limited by the lack of reproducibility of specific laboratory tests over a long term (several months-years), the use of drugs required for other indications, and selection bias of the patients recruited compared with real-life patient groups (eg, TNFi are not used often in patients with heart failure, the clinical setting where most thrombotic events accumulate).

In summary, the study by Manfredi $e t a l,{ }^{1}$ together with evidence from other sources, demonstrate clearly that the axis of megakaryopoiesis, circulating platelets and platelet cellular complexes, and its modulation by antirheumatic therapies, may be very important in the pathogenic mechanisms of rheumatoid synovitis and related CV comorbidity. Despite the many challenges, it merits much more research attention than it has been receiving thus far.

Contributors AYG wrote the first draft of the manuscript. GDK revised all parts, added several sections. Both authors approved the final version.

Competing interests None declared.

Provenance and peer review Not commissioned; externally peer reviewed.

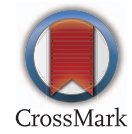

To cite Gasparyan AY, Kitas GD. Ann Rheum Dis 2016;75:1426-1427.

Received 24 February 2016

Revised 10 March 2016

Accepted 19 March 2016

Published Online First 4 April 2016

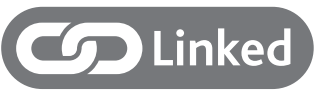

- http://dx.doi.org/10.1136/annrheumdis-2015-208442

Ann Rheum Dis 2016;75:1426-1427.

doi:10.1136/annrheumdis-2015-208720

\section{REFERENCES}

1 Manfredi AA, Baldini M, Camera M, et al. Anti-TNF $\alpha$ agents curb platelet activation in patients with rheumatoid arthritis. Ann Rheum Dis 2016;75: 1511-20.

2 Liu J, DeNofrio J, Yuan W, et al. Genetic manipulation of megakaryocytes to study platelet function. Curr Top Dev Biol 2008;80: 311-35.

3 Lambert MP, Meng R, Xiao L, et al. Intramedullary megakaryocytes internalize released platelet factor 4 and store it in alpha granules. J Thromb Haemost 2015;13:1888-99.

4 Sreeramkumar V, Adrover JM, Ballesteros I, et al. Neutrophils scan for activated platelets to initiate inflammation. Science 2014;346:1234-8.

5 Gasparyan AY, Stavropoulos-Kalinoglou A Mikhailidis DP, et al. Platelet function in rheumatoid arthritis: arthritic and cardiovascular implications. Rheumatol Int 2011;31:153-64.

6 Boilard E, Nigrovic PA, Larabee K, et al. Platelets amplify inflammation in arthritis via collagen-dependent microparticle production. Science 2010:327:580-3.

7 Zhou Q, Xu C, Cheng X, et al. Platelets promote cartilage repair and chondrocyte proliferation via ADP in a rodent model of osteoarthritis. Platelets 2015: Published Online First 1 Sep 2015. doi:10.3109/ 09537104.2015 .1075493$.

8 Alsousou J, Thompson M, Harrison P, et al. Effect of platelet-rich plasma on healing tissues in acute ruptured Achilles tendon: a human immunohistochemistry study. Lancet 2015:385(Suppl 1):S19.

9 Willeke $P$, Kümpers $P$, Schlüter $B$, et al. Platelet counts as a biomarker in ANCA-associated vasculitis. Scand J Rheumatol 2015;44:302-8.

10 Gasparyan AY, Ayvazyan L, Mikhailidis DP, et al. Mean platelet volume: a link between thrombosis and inflammation? Curr Pharm Des 2011;17: 47-58.

11 Fu H, Qin B, Hu Z, et al. Neutrophil- and platelet-tolymphocyte ratios are correlated with disease activity in rheumatoid arthritis. Clin Lab 2015; 61:269-73.

12 Habets KL, Trouw LA, Levarht EW, et al. Anticitrullinated protein antibodies contribute to platelet activation in rheumatoid arthritis. Arthritis Res Ther 2015;17:209.

13 Weinblatt $\mathrm{ME}$, Keystone $\mathrm{EC}$, Furst $\mathrm{DE}$, et al Adalimumab, a fully human anti-tumor necrosis factor alpha monoclonal antibody, for the treatment of rheumatoid arthritis in patients taking concomitant methotrexate: the ARMADA trial. Arthritis Rheum 2003;48:35-45.

14 Matsuno H. Remarkable efficacy of tocilizumab for treating rheumatoid arthritis in patients with high platelet counts. Mod Rheumatol 2015;25: 38-42.

15 Gabay C, Emery P, van Vollenhoven R, et al. Tocilizumab monotherapy versus adalimumab monotherapy for treatment of rheumatoid arthritis (ADACTA): a randomised, double-blind, controlled phase 4 trial. Lancet 2013;381: 1541-50.

16 Zeidler C, Kanz L, Hurkuck F, et al. In vivo effects of interleukin- 6 on thrombopoiesis in healthy and irradiated primates. Blood 1992;80: 2740-5.

17 Lemancewicz D, Bolkun L, Mantur M, et al. Bone marrow megakaryocytes, soluble P-selectin and thrombopoietic cytokines in multiple myeloma patients. Platelets 2014;25:181-7.

18 Nurmohamed MT, Heslinga M, Kitas GD. Cardiovascular comorbidity in rheumatic diseases. Nat Rev Rheumatol 2015;11: 693-704. 